\title{
The psychosocial outcomes of anoxic brain injury following cardiac arrest
}

\author{
Michelle Wilson ${ }^{\mathrm{a}, *}$, Andrew Staniforth ${ }^{\mathrm{b}}$, Richard Till $^{\mathrm{c}}$, Roshan das Nair ${ }^{\mathrm{d}}$, Patrick Vesey ${ }^{\mathrm{e}}$ \\ a Trent Doctorate in Clinical Psychology, University of Lincoln, Hull Community Stroke Team, Highlands Health Centre, Lothian Way, Bransholme, \\ Hull HU7 5DD, United Kingdom \\ b Nottingham University Hospitals NHS Trust, City Hospital Campus, Linby Cardiology, Hucknall Road, Nottingham NG5 1PB, United Kingdom \\ c Basildon and Thurrock NHS Trust, Essex Cardiothoracic Centre, Basildon University Hospital, Nethermayne, Basildon, Essex SS16 5NL, United Kingdom \\ d Institute of Work, Health \& Organisations, University of Nottingham, B09, IWHO, B Floor, International House, Jubilee Campus, Nottingham \\ NG8 1BB, United Kingdom \\ eNottingham University Hospitals NHS Trust, Neurosciences, Queen's Medical Centre, Derby Road, Nottingham NG7 2UH, United Kingdom
}

\author{
A B S T R A C T
}

\begin{abstract}
Aim of the study: This exploratory study aimed to investigate the psychosocial outcomes for cardiac arrest survivors and explore if there is a greater impact on psychosocial outcome for individuals experiencing anoxic brain injury as a result of the cardiac arrest.

Methods: Self-report measures were used to compare the quality of life, social functioning and symptoms of anxiety, depression and posttraumatic stress of individuals with and without anoxic brain injury. Secondary measures of subjective memory and executive difficulties were also used. Fifty-six participants ( 27 with anoxia, 29 without anoxia) took part in the study between six months and four years after experiencing cardiac arrest.

Results: A MANOVA identified a significant difference between the two groups, with the anoxia group reporting more psychosocial difficulties. They reported more social functioning difficulties and more anxiety, depression and post-traumatic stress symptoms. There was, however, no significant difference in self-reported quality of life between the two groups.

Conclusion: As the first known study to compare psychosocial outcomes for cardiac arrest survivors experiencing anoxic brain injury with those without anoxia, the current results suggest that cardiac arrest survivors with subsequent acquired brain injury experience more psychosocial difficulties. This could be due to a combination of neuropsychological, social and psychological factors.
\end{abstract}

\section{Introduction}

As medical interventions advance, the number of people surviving cardiac arrest (CA) is increasing. ${ }^{1} \mathrm{~A}$ lack of oxygen to the brain for just a few minutes during $\mathrm{CA}$ can result in diffuse acquired brain injury $(\mathrm{ABI}){ }^{2}$ In clinical practice the main method of identifying $\mathrm{ABI}$ and predicting outcome is clinical examination. ${ }^{3}$ Clinical examination to identify ABI includes level of consciousness, presence of seizure activity, brainstem reflexes and vestibular reflexes. ${ }^{3,4}$ The Glasgow Comma Scale (GCS) is a commonly used scale to rate the level of consciousness, with coma usually being defined as a GCS of below $8 .{ }^{5}$ The GCS is frequently used as an indicator of severity

$\sim$ A Spanish translated version of the abstract of this article appears as Appendix in the final online version at http://dx.doi.org/10.1016/j.resuscitation.2014.02.008. * Corresponding author.

E-mail address: micmichelle.wilson5@nhs.net (M. Wilson). of brain injury with GCS of 8 or less indicating severe brain injury. ${ }^{6}$ Evidence suggests that GCS score is one of the most reliable predictors of survival and outcome, with lower scores being associated with poor survival rates and greater disability. ${ }^{7}$ The structural damage to the brain caused by anoxia can vary, with areas particularly susceptible being the cerebral cortex, hippocampus, cerebellum and basal ganglia. ${ }^{8}$ Measures of the effectiveness of medical interventions for CA have tended to focus on survival rates, however there is an increasing emphasis on the importance of exploring the functional and psychosocial outcome for CA survivors.

The term 'psychosocial' describes a broad range of psychological and social functions. Psychosocial outcomes have been widely investigated in health conditions, including neurological and cardiac disorders, however the psychosocial outcomes of ABI following $\mathrm{CA}$ is an under-researched area, with much of the psychological research concentrating on cognitive outcomes.9,10

The limited existing research has provided variable findings; however there are suggestions that individuals have lower quality 
of life (QOL) when compared to the general population.11-13 Survivors of CA with ABI have also been found to experience psychological difficulties including anxiety, ${ }^{14}$ depression ${ }^{14,15}$ and post-traumatic stress disorder (PTSD) symptoms. ${ }^{14,16}$ Difficulties with social functioning ${ }^{14,17}$ have also been identified.

No identified study has explored whether the resulting ABI following CA results in more psychosocial difficulties. The aim of the current exploratory study was to investigate the psychosocia outcomes for CA survivors, specifically assessing whether there is a greater impact on psychosocial outcomes in individuals experiencing $\mathrm{ABI}$.

\section{Method}

\subsection{Participants}

Individuals who had experienced out-of-hospital CA between six months and four years previously were included in the study. Categorization of the groups (anoxia vs. non-anoxia) was based on the documentation of clinical decisions at the time of CA. Participants in the anoxia group had been identified as experiencing probable $\mathrm{ABI}$ at the time of $\mathrm{CA}$ based on clinical symptoms (e.g. GCS < 8). These individuals were admitted to ITU for a period of therapeutic hypothermia to reduce cerebral damage. Participants in the non-anoxia group were those with a clinical presentation at the time of CA that suggested no ABI (e.g. GCS > 8). A main indication of neurological damage as a result of anoxia is coma. ${ }^{3}$

All participants were aged over 18 and were English-speaking. All participants had been treated with an implantable cardioverter defibrillator (ICD). Individuals with other medical diagnosis or history that would affect neuropsychological functioning, or premorbid psychological difficulties (as identified from the medical notes and on self-report) were excluded from the study. The two groups were compared using a range of psychosocial measures including QOL, social functioning and symptoms of anxiety, depression and PTSD. Measures of subjective memory and executive difficulties were also included as previous findings have suggested psychosocial difficulties are associated with cognitive difficulties ${ }^{11,17}$ which may result from ABI.

\subsection{Measures}

\subsubsection{Quality of life}

QOL was measured using the Quality of Life Scale (QOLS), ${ }^{18}$ a 16-item questionnaire where individuals rate their satisfaction in relation to a range of items including daily and social activities and relationships.

The QOLS has been adapted for use with individuals with health conditions and was found to be useful with a range of chronic health conditions, including cardiac conditions. ${ }^{19}$

\subsubsection{Social functioning}

The Social Functioning Questionnaire $(\mathrm{SFQ})^{20}$ was used as a measure of perceived social functioning. The SFQ is a reliable and valid $^{21}$ eight-item questionnaire on which individuals rate their functioning in areas including work and home tasks, finances, relationships and social activities.

\subsubsection{Depression and anxiety symptoms}

Symptoms of depression and anxiety were measured using the 14-item Hospital Anxiety and Depression Scale (HADS). ${ }^{22}$ The HADS has good reliability and validity when assessing anxiety and depression in medical patients. ${ }^{23}$

\subsubsection{Post-traumatic stress symptoms}

PTSD symptoms were measured using the Impact of Event ScaleRevised (IES-R). ${ }^{24}$ This 22 -item questionnaire is based on the original Impact of Event Scale ${ }^{25}$ with the introduction of seven new items exploring hyper-arousal symptoms, as well as avoidance and intrusion symptoms ${ }^{26}$ This measure has been used with cardiac patients $^{22}$ and has good reliability, ${ }^{27}$ internal consistency, ${ }^{28,29}$ and concurrent and discriminant validity. ${ }^{28}$

\subsubsection{Subjective rating of memory difficulties}

A subjective rating of everyday memory failures was obtained using the 13-item Everyday Memory Questionnaire-Revised (EMQ$\mathrm{R})^{30}$ adapted from the original Everyday Memory Questionnaire. ${ }^{31}$

\subsubsection{Subjective rating of executive difficulties}

A subjective rating of executive difficulties was obtained using the Dysexecutive Questionnaire (DEX) from the Behavioural Assessment of the Dysexecutive Syndrome. ${ }^{32}$ The questionnaire was completed by the participants and an independent rater. The 20 items consider changes to emotion or personality, motivation, cognition, and behaviour.

\subsection{Method}

Ethical approval was obtained from the Derbyshire Research Ethics Committee. Participants were identified from existing databases of ICD patients from two UK NHS Trusts. Individuals meeting the initial inclusion criteria were sent the questionnaires by post.

The measures were scored in accordance with the standardised instructions and any missing data was replaced with the participant's mean score for the scale or subscale. The scores of the two groups were compared using multiple analyses of variance (MANOVA).

\subsection{Participant characteristics}

Of the 168 individuals invited to participate in the study, 64 returned the completed questionnaires (38\% response rate). Eight individuals were excluded from the study due to a pre-CA history of mental health problems or other neurological diagnoses. Therefore a total of 56 participants were included in the study, 27 $(48 \%)$ of whom met the criteria for probable anoxia and $29(52 \%)$ for non-anoxia.

Participants' ages ranged from 37 to 84 years (mean $=66.13$, $\mathrm{SD}=12.61)$ and these were similar across the two groups. Details of participant characteristics can be seen in Table 1. Participants in the two groups were well matched in regard to time since CA, with the mean time being 27.78 months for the anoxia group and 25.81 months for the non-anoxia group.

\section{Results}

A MANOVA was used to assess the differences between the anoxia and non-anoxia groups across the range of psychosocial outcome measures. The IES-R scores were not included in the MANOVA as they violated the assumption of normality. There was a significant multivariate difference between the two groups (Pil-lai's Trace $=0.172, f(4,51)=2.656, p=0.043$; effect size $\left(\right.$ Partial eta squared $\left.\left[p^{2}\right]\right)=0.172$, observed power $\left.=0.700\right)$. The mean scores on the measures for both groups and the results of the univariate tests are in Table 2

The anoxia group reported significantly more anxiety symptoms $(f(1)=4.959, p=0.030$, Cohen's $d=0.59)$, depression symptoms $(f(1)=5.857, p=0.019$, Cohen's $d=0.64)$ and significantly more difficulties with social functioning $(f(1)=5.873, p=0.019$, Cohen's $d=0.64$ ) compared to the non-anoxia group. There was no significant difference between the two groups on the QOLS $(f(1)=0.659$, 
Table 1

Participant characteristics.

\begin{tabular}{|c|c|c|c|}
\hline & All participants $(n=56)$ & Anoxia $(n=27)$ & Non-anoxia $(n=29)$ \\
\hline Age, mean (SD) & $66.13(12.61)$ & $63.96(12.59)$ & $67.56(12.64)$ \\
\hline \multicolumn{4}{|l|}{ Gender } \\
\hline Male & $37(66 \%)$ & $13(48 \%)$ & $24(83 \%)$ \\
\hline Female & $19(35 \%)$ & $14(52 \%)$ & $5(17 \%)$ \\
\hline \multicolumn{4}{|l|}{ Living arrangements } \\
\hline Living alone & $8(14 \%)$ & $2(7 \%)$ & $6(21 \%)$ \\
\hline Living with partner/family & $46(82 \%)$ & $24(89 \%)$ & $22(76 \%)$ \\
\hline With carer & $1(2 \%)$ & $1(4 \%)$ & 0 \\
\hline No information & $1(2 \%)$ & 0 & $1(3 \%)$ \\
\hline \multicolumn{4}{|l|}{ Employment status } \\
\hline Full-time employment & $9(16 \%)$ & $4(15 \%)$ & $5(17 \%)$ \\
\hline Part-time employment & $5(9 \%)$ & $3(11 \%)$ & $2(7 \%)$ \\
\hline Retired & $39(69 \%)$ & $16(59 \%)$ & $23(79 \%)$ \\
\hline Unemployed & $1(2 \%)$ & 0 & $1(3 \%)$ \\
\hline Sick leave & $1(2 \%)$ & $1(4 \%)$ & 0 \\
\hline Voluntary work & $1(2 \%)$ & $1(4 \%)$ & 0 \\
\hline Time since CA (months) & $26.80(12.87)$ & $27.78(12.99)$ & $25.81(12.91)$ \\
\hline \multicolumn{4}{|l|}{ Mean (SD) } \\
\hline \multicolumn{4}{|l|}{ Bystander CPR } \\
\hline Yes & $41(73 \%)$ & $20(74 \%)$ & $21(72 \%)$ \\
\hline No & $10(18 \%)$ & $5(19 \%)$ & $5(17 \%)$ \\
\hline No information & $5(9 \%)$ & $2(7 \%)$ & $3(10 \%)$ \\
\hline GCS score Mean (SD) & & $4.62(4.16)$ & $12.19(4.90)$ \\
\hline
\end{tabular}

$p=0.420$, Cohen's $d=0.2$ ). A Mann-Whitney test identified a significant difference between the groups on the IES-R; with the anoxia group reporting more PTSD symptoms than the nonanoxia group (total score, $u=237.0, p=0.011$; avoidance, $u=$ $236.5, p=0.011$; intrusion, $u=254.5, p=0.024$; hyperarousal, $u$ $=237.5, p=0.010$ ).

The number of participants exceeding clinical cut-off scores on the measures can be seen in Table 3. Fifty-two percent of participants in the anoxia group scored above the cut-off score on the anxiety scale of the HADS, with $11 \%$ of this group meeting the criteria for severe anxiety. A third of the anoxia group also reported experiencing mild or moderate levels of depression, compared to $7 \%$ of the non-anoxia group. In regard to the IES-R, $22 \%$ of the anoxia group had scores representing clinical levels of PTSD symptoms compared to $7 \%$ of the non-anoxia group.

As psychological distress and poor social functioning are associated with reduced QOL, it would be expected that the anoxia group would report significantly lower QOL. This was not the case in the current study however, as there was no significant difference in scores on the QOLS between both groups. Consistent with previous findings, ${ }^{33}$ however, poorer QOL was associated with more anxiety symptoms $(r=-0.690, p>0.001)$, depression symptoms $(r=-0.767$, $p>0.001)$, PTSD symptoms $(r \mathrm{~s}=-0.623, p=0.001)$ and more social functioning difficulties $(r=-0.710, p>0.001)$ for the anoxia group. Poorer QOL was also associated with more anxiety symptoms $(r=$ $-0.520, p=0.004)$, depression symptoms $(r=-0.527, p=0.003)$ and more social functioning difficulties $(r=-0.566, p=0.001)$ for the non-anoxia group.

There was no statistically significant difference between the two groups on subjective memory difficulties (total score, $t(54)=1.313$, $p=0.195)$, or self-rated executive difficulties $(t(56)=0.857, p=$ $0.395)$. There were significant correlations between the scores on the psychosocial measures and the scores on both the EMQ-R and DEX (see Table 4). For the anoxia group more subjective memory difficulties were significantly associated with more social functioning difficulties, poorer QOL and anxiety, depression and PTSD symptoms. More self-reported executive difficulties were significantly associated with more difficulties on all of the measures other than PTSD symptoms. For the non-anoxia group more subjective memory and executive function difficulties were associated

Table 2

Mean scores and standard deviations of measures.

\begin{tabular}{|c|c|c|c|c|}
\hline Measure & All participants & Anoxia & Non-anoxia & Difference $(p)$ \\
\hline QOLS & $84.71(14.87)$ & $83.04(17.04)$ & $86.23(12.63)$ & 0.420 \\
\hline SFQ & $4.45(3.45)$ & $5.56(3.84)$ & $3.41(2.72)$ & $0.019^{*}$ \\
\hline \multicolumn{5}{|l|}{ HADS } \\
\hline Total & $10.05(7.07)$ & $12.44(8.12)$ & $7.83(5.13)$ & $0.013^{*}$ \\
\hline Anxiety & $6.05(4.42)$ & $7.37(4.88)$ & $4.83(3.61)$ & $0.030^{*}$ \\
\hline Depression & $3.98(3.28)$ & $5.04(3.78)$ & $3.00(2.43)$ & $0.019^{*}$ \\
\hline \multicolumn{5}{|l|}{ IES-R } \\
\hline Total & $18.25(19.50)$ & $25.63(23.11)$ & $11.38(12.21)$ & $0.011^{*}$ \\
\hline Avoidance & $0.88(0.93)$ & $1.20(1.07)$ & $0.57(0.66)$ & $0.011^{*}$ \\
\hline Intrusion & $0.86(0.95)$ & $1.21(1.14)$ & $0.54(0.60)$ & $0.024^{*}$ \\
\hline Hyper-arousal & $0.76(0.97)$ & $1.11(1.19)$ & $0.44(0.59)$ & $0.010^{*}$ \\
\hline \multicolumn{5}{|l|}{ EMQ-R } \\
\hline Total & $13.30(13.08)$ & $15.67(13.85)$ & $11.10(12.14)$ & 0.195 \\
\hline Retrieval & $1.27(1.14)$ & $1.44(1.19)$ & $1.10(1.08)$ & 0.258 \\
\hline Attentional tracking & $0.78(0.96)$ & $1.00(1.09)$ & $0.57(0.79)$ & 0.093 \\
\hline \multicolumn{5}{|l|}{ DEX } \\
\hline Self & $15.32(10.97)$ & $17.93(12.10)$ & $12.90(9.37)$ & 0.087 \\
\hline Independent & $13.23(11.43)$ & $14.60(14.02)$ & $11.97(8.40)$ & 0.395 \\
\hline
\end{tabular}

* Statistically significant.

ANOVA test used for QOLS, SFQ and HADS; Mann-Whitney U test used for IES-R; T-test used for EMQ-R and DEX. 
Table 3

Number and percentage of participants scoring within the clinical range.

\begin{tabular}{|c|c|c|c|}
\hline Measure (cut-off score) & All participants & Anoxia & Non-anoxia \\
\hline \multicolumn{4}{|l|}{ HADS-depression } \\
\hline Mild (8-10) & $9 \quad(16 \%)$ & $7 \quad(26 \%)$ & $2(7 \%)$ \\
\hline Moderate (11-15) & $2(4 \%)$ & $2(7 \%)$ & $0(0 \%)$ \\
\hline Severe $(>16)$ & $0 \quad(0 \%)$ & $0 \quad(0 \%)$ & $0(0 \%)$ \\
\hline Total $(>8)$ & $11(19 \%)$ & $9 \quad(33 \%)$ & $2(7 \%)$ \\
\hline \multicolumn{4}{|l|}{ HADS-anxiety } \\
\hline Mild (8-10) & $13(23 \%)$ & $9 \quad(33 \%)$ & $4(14 \%)$ \\
\hline Moderate (11-15) & $4 \quad(7 \%)$ & $2(7 \%)$ & $2(7 \%)$ \\
\hline Severe $(>16)$ & $3(5 \%)$ & $3 \quad(11 \%)$ & $0(0 \%)$ \\
\hline Total $(>8)$ & $20(36 \%)$ & $14(52 \%)$ & $6(21 \%)$ \\
\hline \multicolumn{4}{|l|}{ IES-R } \\
\hline Total $(>33)$ & $8 \quad(14 \%)$ & $6 \quad(22 \%)$ & $2(7 \%)$ \\
\hline Avoidance $(>1.5)$ & $11(20 \%)$ & $8 \quad(30 \%)$ & $3(10 \%)$ \\
\hline Intrusion $(>1.5)$ & $11(20 \%)$ & $9 \quad(33 \%)$ & $2(7 \%)$ \\
\hline Hyper-arousal (>1.5) & $10(18 \%)$ & $7 \quad(26 \%)$ & $3(10 \%)$ \\
\hline \multicolumn{4}{|l|}{ EMQ-R } \\
\hline Total (>2.07) & $8 \quad(14 \%)$ & $4 \quad(15 \%)$ & $4(14 \%)$ \\
\hline Retrieval (>2.68) & $9 \quad(16 \%)$ & $5 \quad(19 \%)$ & $4(14 \%)$ \\
\hline Attentional (>1.89) & $5(9 \%)$ & $3 \quad(11 \%)$ & $2(7 \%)$ \\
\hline
\end{tabular}

HADS (Hospital Anxiety and Depression Scale) cut-off scores suggested by Snaith and Zigmond. ${ }^{34}$

IES-R (Impact of event scale-revised) cut-off scores suggested by Creamer et al. ${ }^{29}$ EMQ-R (Everyday Memory Questionnaire) cut-off scores suggested by Royle and Lincoln. ${ }^{30}$

Table 4

Associations between psychosocial outcome and subjective cognitive difficulties.

\begin{tabular}{lcccl} 
& \multicolumn{2}{c}{ Anoxia group } & \multicolumn{2}{c}{ Non-anoxia group } \\
\cline { 2 - 5 } & DEX (self) & EMQ-R & DEX (self) & EMQ-R \\
\hline SFQ & $0.156^{* *}$ & $0.460^{*}$ & $0.882^{* *}$ & $0.617^{* *}$ \\
Depression & $0.560^{* *}$ & $0.560^{* *}$ & 0.350 & 0.336 \\
Anxiety & $0.627^{* *}$ & $0.773^{* *}$ & $0.569^{* *}$ & $0.427^{*}$ \\
QOLS & $-0.487^{* *}$ & $-0.401^{* *}$ & $-0.533^{* *}$ & $0.455^{* *}$ \\
IES & 0.374 & $0.425^{*}$ & $0.656^{* *}$ & $0.523^{* *}$ \\
\hline
\end{tabular}

* Significant to 0.05 level. **

Significant to 0.01 level.

with more social functioning difficulties, poorer QOL and more anxiety and PTSD symptoms, but not depression symptoms.

For the anoxia group, only scores on the anxiety subscale of the HADS were significantly associated with age, with younger participants reporting more difficulties (see Table 5). For the non-anoxia group age was significantly associated with scores on the SFQ and IES-R, again with younger participants reporting more difficulties. There were no statistically significant gender differences for any of the measures and none of the scores were associated with the length of time since CA.

\section{Discussion}

This study identified that individuals who experienced anoxia as a result of CA experienced significantly more psychosocial difficulties than individuals who experienced CA without anoxia. Individuals who had anoxia reported significantly more social functioning difficulties and anxiety, depression and PTSD symptoms. As well as adjusting to life following CA, and the social and health implications of this, the anoxia group also have to adjust to living with $\mathrm{ABI}$.

Living with $\mathrm{ABI}$ can result in reduced activities and social contact due to cognitive difficulties and increased dependence. ${ }^{14}$ This was consistent with findings that individuals with anoxia reported significantly more social functioning difficulties compared to the non-anoxia group. Also consistent with previous research findings, ${ }^{17}$ social functioning difficulties were significantly associated with subjective memory and executive difficulties.

Anxiety was a common problem experienced by the anoxia group, with $52 \%$ having at least mild clinical symptoms. In the nonanoxia group, $21 \%$ of participants experienced clinical levels of anxiety. Snaith and Zigmond (1994) ${ }^{34}$ reported that $20.6 \%$ of a nonclinical population scored within the mild range on the HADS. In the current study, more participants in the anoxia group than would be expected scored within this range (33\%); and in the non-anoxia group, $14 \%$ scored within this range. Seven percent of both groups scored within the moderate anxiety range, compared to $10 \%$ of the general population. The percentage of the general population scoring within the severe anxiety range was $2.6 \%$, none of the nonanoxia group met this level of symptoms, however $11 \%$ of the anoxia group did, which is more than would be expected based on the findings from the general population.

Mild and moderate depression symptoms were identified in $33 \%$ of the participants with anoxia compared to $7 \%$ in the non-anoxia group. More of the anoxia group (26\%) scored at the mild depression level compared to findings from a non-clinical general population ${ }^{34}$ (7.8\%) and a similar proportion of the non-anoxia group (7\%) did Seven percent of the anoxia group and none of the non-anoxia group scored within the moderate depression range compared to $2.9 \%$ of the general population.

From a neuropsychological perspective, the emotional consequence could be a result of the specific neurological damage sustained. Areas of the brain associated with anxiety include the hippocampus and cingulate and pre-frontal cortex ${ }^{35}$ and depression has been found to be associated with damage to the left dorsolateral frontal areas and the basal ganglia. ${ }^{36}$ These brain regions have also been identified to be particularly susceptible to anoxic damage.

The social implications of CA and ABI, such as reduced activities and social functioning, and how individuals appraise their

Table 5

Statistical results for gender differences and association with age and time since CA.

\begin{tabular}{|c|c|c|c|c|c|c|}
\hline \multirow[t]{2}{*}{ Measure } & \multicolumn{2}{|c|}{ Gender differences } & \multicolumn{2}{|c|}{ Association with age } & \multicolumn{2}{|c|}{ Association with time since CA } \\
\hline & Anoxia & Non-anoxia & Anoxia & Non-anoxia & Anoxia & Non-anoxia \\
\hline QOLS & $t=0.728$ & $t=-0.523$ & $r=0.200$ & $r=0.065$ & $\bar{r}=-0.086$ & $r=0.016$ \\
\hline SFQ & $\mathrm{t}=-0.022$ & $\mathrm{t}=0.914$ & $\mathrm{r}=-0.106$ & $r=-0.452^{*}$ & $\mathrm{r}=-0.005$ & $\mathrm{r}=-0.145$ \\
\hline EMQ-R & $\mathrm{t}=0.145$ & $t=0.621$ & $\mathrm{r}=-0.252$ & $\mathrm{r}=0.109$ & $\mathrm{r}=-0.277$ & $\mathrm{r}=0.029$ \\
\hline HADS (D) & $t=0.763$ & $\mathrm{t}=0.198$ & $\mathrm{r}=-0.041$ & $\mathrm{r}=0.079$ & $\mathrm{r}=0.092$ & $\mathrm{r}=-0.002$ \\
\hline HADS (A) & $t=0.768$ & $\mathrm{t}=0.421$ & $r=-0.437^{*}$ & $r=-0.172$ & $r=-0.009$ & $\mathrm{r}=-0.004$ \\
\hline $\operatorname{DEX}(\mathrm{S})$ & $\mathrm{t}=1.026$ & $t=0.648$ & $\mathrm{r}=-0.060$ & $\mathrm{r}=-0.322$ & $\mathrm{r}=-0.090$ & $\mathrm{r}=-0.100$ \\
\hline $\operatorname{DEX}(\mathrm{I})$ & $t=1.623$ & $\mathrm{t}=1.230$ & $\mathrm{r}=-0.045$ & $\mathrm{r}=-0.016$ & $r=-0.333$ & $\mathrm{r}=-0.099$ \\
\hline IES-R & $\mathrm{u}=69.00$ & $\mathrm{u}=56.00$ & $\mathrm{rs}=-0.350$ & $\mathrm{rs}=-0.391^{*}$ & $\mathrm{rs}=-0.128$ & $\mathrm{rs}=-0.152$ \\
\hline
\end{tabular}

* Statistically significant.

SFQ, social Functioning Questionnaire: IES-R, Impact of Event Scale-Revised; QOLS, Quality of Life Scale; HADS (D). Hospital Anxiety and Depression Scale depression subscale; HADS (A), Hospital Anxiety and Depression Scale anxiety subscale; EMQ-R (T), Everyday Memory Questionnaire-revised (total score), DEX (s), Dysexecutive Questionnaire (self-rater), DEX (I), independent-rater. 
situation, may also impact on their mood. Individuals with cognitive difficulties that affect their ability to undertake activities may perceive their ability to cope as poor and see activities as a threat. Individuals who regularly perceive threat and inability to cope can experience anxiety. This may result in the avoidance of activities which maintains the anxiety. Those who perceive their situation as one of loss may be more susceptible to low mood. Perceiving loss of ability to return to how they were prior to the CA as a failure can further result in avoiding activities.

Clinically significant PTSD symptoms were present in $22 \%$ of the anoxia group compared to $7 \%$ of the non-anoxia group. The experience of treatment on ITU for individuals in the anoxia group could have contributed to the development of PTSD symptoms. Evidence suggests that between $14 \%$ and $27 \%$ of individuals admitted to ICUs experience PTSD symptoms. ${ }^{37}$ Individuals who appraise their time in ITU as a traumatic and unpleasant experience which was a significant threat to their life, as opposed to those who appraise it as a medical intervention that saved their life, may be more likely to experience subsequent PTSD symptoms.

Despite the anoxia group having significantly more psychosocial difficulties than the non-anoxia group, there was no significant difference in scores on the QOLS between the two groups. Consistent with previous findings ${ }^{10}$ however, poorer QOL was found to be associated with more social functioning difficulties, more anxiety, depression and PTSD symptoms and more subjective cognitive difficulties.

Previous studies have found that survivors of CA have significantly lower QOL compared to non-clinical populations. ${ }^{11-13}$ The mean score for both the anoxia (83.04) and non-anoxia (86.23) groups was below the non-clinical population average of $90,{ }^{19}$ with $70 \%$ of the anoxia group and $62 \%$ of the non-anoxia group scoring below the general population mean.

Consistent with previous research, ${ }^{11,17}$ greater psychosocial difficulties were found to be associated with more self-reported cognitive difficulties. Despite this, however, there was no significant difference between the two groups with regard to selfreported memory and executive difficulties. A possible explanation for this is that individuals in the anoxia group may have lacked insight into their cognitive difficulties which can be common following $\mathrm{ABI}^{36}$ This is associated with poor psychosocial functioning as individuals with poor insight have high expectations of their ability and recovery; when they fail to complete activities as they had prior to the ABI, they perceive failure and experience distress.

Psychosocial outcomes can be used to assess the effectiveness of medical interventions for CA. Psychosocial difficulties have also been found to impact on patients' health. ${ }^{38,39}$ The current findings suggest that CA survivors, particularly those who experience anoxia, should receive assessment and support to facilitate adaptive adjustment. Health professionals regularly involved in patients' care, such as Cardiologists and General Practitioners (GPs), should be aware of the increased risk of psychological and social difficulties for this population and be familiar with the symptoms. CA survivors should also be provided with information about this risk and encouraged to discuss their symptoms. Psycho-education about the impact of avoidance of activity and the value of social support and appropriate coping strategies could be beneficial. Cardiologists and GPs could support this by promoting activity and providing recommendations to patients and carers about types and levels of activity they can still engage in. Individuals who develop psychological difficulties such as PTSD, anxiety or depression should be referred to psychological services as appropriate. Individuals who experience ABI should also receive support for their cognitive difficulties. This should include neuropsychologi-cal assessment to identify specific deficits and to guide cognitive rehabilitation.
Limitations of the current study include the retrospective design and relatively small sample size. We acknowledge that not all postcardiac arrest patients have an ICD, and therefore the participants are not necessarily representative of all cardiac arrest patients. However, with technological advances, and the use of ICDs as secondary prevention being recommended by health guidance, more people today are fitted with ICDs than ever before. Despite these limitations, the findings of this exploratory study provide a basis for further research into the outcome for CA survivors.

A number of interacting neuropsychological, social and psychological factors could result in the psychosocial difficulties experienced and future research could further explore the factors involved. For example, neuro-imaging studies could investigate the areas of the brain affected in CA survivors with ABI and whether there is an association between neurological damage and psychoso-cial functioning.

Although the interest of the current study was of individuals' subjective cognitive difficulties, further research could combine subjective and objective measures of cognitive functioning to explore the association between cognitive difficulties and psy-chosocial difficulties. This could also explore whether lack of insight may be involved in the emergence of difficulties. Individuals' level of dependence, coping strategies, level of activity and level of social support could all be used to further examine the factors involved in the psychosocial difficulties resulting from $\mathrm{ABI}$ following $\mathrm{CA}$.

\section{Conclusion}

The current findings add to the limited literature exploring the psychosocial outcomes of anoxia following CA. As the first known study to compare outcome for CA survivors experiencing ABI with those without, the results suggest that $\mathrm{CA}$ survivors with subsequent $\mathrm{ABI}$ experience more psychosocial difficulties. Individuals with $\mathrm{ABI}$ reported significantly more social functioning difficulties and symptoms of anxiety, depression and PTSD. Although better QOL was associated with fewer psychosocial difficulties, there was no statistically significant difference in subjective QOL between the two groups. A combination of neuropsychological, social and psychological factors resulting from ABI following CA may explain this difference; however further research is required to explore this in more depth.

\section{Conflict of interest statement There}

are no conflicts of interest.

\section{References}

1. Hirsch KG, Koenig MA, Geocadin RG. Management of brain injury after cardiac arrest. CONTINUUM: Lifelong Learn Neurol 2009;15:100-20.

2. Cohan SL, Mun SK, Petite J, Correia J, Tavelra Da Silva AT, Waldhorn RE. Cerebral blood flow in humans following resuscitation from cardiac arrest. Stroke 1989;20:761-5

3. Nolan JP, Neumar RW, Adrie C, et al. Post-cardiac arrest syndrome: epidemiology, pathophysiology, treatment, and prognostication: a scientific statement from the international liaison committee on resuscitation; the American Heart Association Emergency Cardiovascular Care Committee; the council on cardiovascular surgery and anesthesia; the council on cardiopulmonary, perioperative, and critical care; the council on clinical cardiology; the council on stroke. Resuscitation 2008;79:350-79.

4. Meyer N, Hall J. Bench-to-bedside review: brain dysfunction in critically ill patients - the intensive care unit and beyond. Crit Care 2006;10:223.

5. Jennett B. Development of Glasgow coma and outcome scales. Nepal J Neurosci 2005;2:24-8

6. Balestreri M, Czosnyka M, Chatfield DA, et al. Predictive value of Glasgow Coma Scale after brain trauma: change in trend over the past ten years. J Neurol Neu-rosurg Psychiatry 2004;75:161-2.

7. Schefold JC, Storm C, Krüger A, Ploner CJ, Hasper D. The Glasgow coma score is a predictor of good outcome in cardiac arrest patients treated with therapeutic hypothermia. Resuscitation 2009;80:658-61

Garcia-Molina A, Roig-Rovira T, Ensenat-Cantallops A, et al. Neuropsychological profile of persons with anoxic brain injury: differences regarding physiopathological mechanism. Brain Inj 2006;20:1139-45. 
9. Caine D, Watson JDG. Neuropsychological and neuropathological sequelae of cerebral anoxia: a critical review. J Int Neuropsychol Soc 2000;6:86-99.

10. Moulaert VRMP, Verbunt JA, van Heugten CM, Wade DT. Cognitive impairments in survivors of out-of-hospital cardiac arrest: a systematic review. Resuscitation 2009:80:297-305.

11. Sunnerhagen KS, Johansson O, Herlitz J, Grimby G. Life after cardiac arrest; a retrospective study. Resuscitation 1996;31:135-40.

12. Saner H, Borner Rodriguez E, Kummer-Bangerter A, Schüppel R, von Planta M. Quality of life in long-term survivors of out-of-hospital cardiac arrest. Resuscitation 2002;53:7-13.

13. Lundgren-Nilsson A, Rosén H, Hofgren C, Sunnerhagen KS. The first year after successful cardiac resuscitation: function, activity, participation and quality of life. Resuscitation 2005;66:285-9.

14. Wachelder EM, Moulaert V, van Heugten C, Verbunt JA, Bekkers S, Wade DT. Life after survival: long-term daily functioning and quality of life after an out-of-hospital cardiac arrest. Resuscitation 2009;80:517-22.

15. Cronberg T, Lilja G, Rundgren M, Friberg H, Widner H. Long-term neurological outcome after cardiac arrest and therapeutic hypothermia. Resuscitation 2009;80:1119-23.

16. Ladwig KH, Schoefinius A, Dammann G, Danner R, Gurtler R, Herrmann R Long-acting psychotraumatic properties of a cardiac arrest experience. Am J Psychiatry 1999;156:912.

17. Middelkamp W, Moulaert VRMP, Verbunt JA, van Heugten CM, Bakx WG, Wade DT. Life after survival: long-term daily life functioning and quality of life of patients with hypoxic brain injury as a result of a cardiac arrest. Clin Rehabil 2007;21:425.

18. Burckhardt CS, Woods SL, Schultz AA, Ziebarth DM. Quality of life of adults with chronic illness: a psychometric study. Res Nurs Health 1989;12:347-54.

19. Burckhardt CS, Anderson KL. The quality of life scale (QOLS): reliability, validity, and utilization. Health Qual Life Out 2003;1:60.

20. Tyrer P. Personality disorder and social functioning. In: Measuring human problems: a practical guide; 1990 . p. 119-42.

21. Tyrer P, Nur U, Crawford M, et al. The social functioning questionnaire: a rapid and robust measure of perceived functioning. Int J Soc Psychiatry 2005;51:265.

22. Zigmond AS, Snaith RP. The hospital anxiety and depression scale. Acta Psychiatr Scand 1983;67:361.

23. Herrmann C. International experiences with the hospital anxiety and depression scale - a review of validation data and clinical results. J Psychosom Res $1997 ; 42: 17-41$
Weiss DS, Marmar CR. The impact of events scale - revised. In: Wilson JP, Keane TM, editors. Assessing psychological trauma and PTSD: a handbook for practitioners. New York: Guilford Press; 1997. p. 399-411.

25. Horowitz M, Wilner N, Alvarez W. Impact of event scale: a measure of subjective stress. Psychosom Med 1979;41:209.

26. Weiss D. The impact of event scale: revised. In: Cross-cultural assessment of psychological trauma and PTSD; 2007. p. 219-38.

27. Baumert J, Simon H, Gündel H, Schmitt C, Ladwig KH. The impact of even scale - revised: evaluation of the subscales and correlations to psychophysiological startle response patterns in survivors of a life-threatening cardiac event: an analysis of 129 patients with an implanted cardioverter defibrillator. J Affect Disord 2004;82:29-41.

28. Beck JG, Grant DMM, Read JP, et al. The impact of event scale-revised: psychometric properties in a sample of motor vehicle accident survivors. J Anxiety Disord 2008;22:187-98.

29. Creamer M, Bell R, Failla S. Psychometric properties of the impact of event scale - revised. Behav Res Ther 2003;41:1489-96.

30. Royle J, Lincoln NB. The everyday memory questionnaire-revised development of a 13-item scale. Disabil Rehabil 2008;30:114-21.

31. Sunderland A, Harris JE, Baddeley AD. Do laboratory tests predict everyday memory? A neuropsychological study. J Verbal Learning Verbal Behav 1983;22:341-57.

32. Burgess PW, Alderman N, Wilson BA, Evans JJ, Emslie H. The dysexecutive questionnaire. In: Behavioural assessment of the dysexecutive syndrome. Bury St. Edmunds, UK: Thames Valley Test Company; 1996.

33. Moulaert VRMP, Wachelder EM, Verbunt JA, Wade DT, van Heugten CM. Determinants of quality of life in survivors of cardiac arrest. J Rehabil Med 2010;42:553-8.

34. Snaith RP, Zigmond AS. The Hospital Anxiety and Depression Scale manual Windsor: NFER, Nelson; 1994

35. Gray JA, McNaughton N. The neuropsychology of anxiety: reprise. Nebr Symp Motiv 1996;43:61-134.

36. Ownsworth TL, Oei TPS. Depression after traumatic brain injury: conceptualization and treatment considerations. Brain Inj 1998;12:735-51.

37. Rattray JE, Hull AM. Emotional outcome after intensive care: literature review. J Adv Nurs 2008;64:2-13

38. Barth J, Schumacher M, Herrmann-Lingen C. Depression as a risk factor for mortality in patients with coronary heart disease: a meta-analysis. Psychosom Med 2004;66:802

39. Penninx BWJH, Beekman ATF, Honig A, et al. Depression and cardiac mortality: results from a community-based longitudinal study. Arch Gen Psychiatry 2001;58:221 